\title{
Transcriptomic characterization of cold acclimation in larval zebrafish
}

\author{
Yong Long ${ }^{1}$, Guili Song ${ }^{1}$, Junjun Yan ${ }^{1,2}$, Xiaozhen $\mathrm{He}^{1,2}$, Qing $\mathrm{Li}^{1}$ and Zongbin Cui ${ }^{*}$
}

\begin{abstract}
Background: Temperature is one of key environmental parameters that affect the whole life of fishes and an increasing number of studies have been directed towards understanding the mechanisms of cold acclimation in fish. However, the adaptation of larvae to cold stress and the cold-specific transcriptional alterations in fish larvae remain largely unknown. In this study, we characterized the development of cold-tolerance in zebrafish larvae and investigated the transcriptional profiles under cold stress using RNA-seq.

Results: Pre-exposure of $96 \mathrm{hpf}$ zebrafish larvae to cold stress $\left(16^{\circ} \mathrm{C}\right)$ for $24 \mathrm{~h}$ significantly increased their survival rates under severe cold stress $\left(12^{\circ} \mathrm{C}\right)$. RNA-seq generated 272 million raw reads from six sequencing libraries and about $92 \%$ of the processed reads were mapped to the reference genome of zebrafish. Differential expression analysis identified 1,431 up- and 399 down-regulated genes. Gene ontology enrichment analysis of cold-induced genes revealed that RNA splicing, ribosome biogenesis and protein catabolic process were the most highly overrepresented biological processes. Spliceosome, proteasome, eukaryotic ribosome biogenesis and RNA transport were the most highly enriched pathways for genes up-regulated by cold stress. Moreover, alternative splicing of 197 genes and promoter switching of 64 genes were found to be regulated by cold stress. A shorter isoform of stk 16 that lacks 67 amino acids at the $\mathrm{N}$-terminus was specifically generated by skipping the second exon in cold-treated larvae. Alternative promoter usage was detected for per3 gene under cold stress, which leading to a highly up-regulated transcript encoding a truncated protein lacking the C-terminal domains.
\end{abstract}

Conclusions: These findings indicate that zebrafish larvae possess the ability to build cold-tolerance under mild low temperature and transcriptional and post-transcriptional regulations are extensively involved in this acclimation process.

Keywords: Cold stress, Transcriptomic analysis, Zebrafish, RNA-seq, Gene expression

\section{Background}

Water temperature was suggested to be the master factor that determines nearly all life activities of most fishes, including development, growth, reproduction, metabolism, behavior and geographical distribution [1-3]. Like other ectotherms, fishes may encounter a wide range of daily and seasonal temperature variations in their habitats and deleterious consequences will occur if water temperature exceeds the species-specific thermal tolerance range $[2,4]$. It was reported that most of fish kills in nature are caused by exposure to low temperatures [5]. Thus, the ability to cope with cold stress is quite important for the survival of fishes

\footnotetext{
*Correspondence: zbcui@ihb.ac.cn

${ }^{1}$ The Key Laboratory of Aquatic Biodiversity and Conservation, Institute of Hydrobiology, Chinese Academy of Sciences, Wuhan, Hubei, PR China Full list of author information is available at the end of the article
}

under natural conditions. Furthermore, a large number of commercially important aquaculture species such as tilapia (Oreochromis niloticus), milkfish (Chanos chanos) and red sea bream (Pagrus major) are sensitive to cold stress and mass mortality is often caused by winter cold fronts [6-8]. Therefore, it is of great significance for both scientific researches and fisheries to investigate the mechanisms underlying cold-tolerance of fish.

Since the middle of the 20th century, an increasing number of studies were performed to characterize the acclimation responses of fishes to cold stress. It was revealed that fishes can gradually establish cold adaptive phenotypes through extensive biochemical, metabolic and physiological regulations $[9,10]$. Well-defined biochemical and physiological acclimations include producing temperature-specific isozymes [9], altering the content of membrane lipid and the degree of fatty acid unsaturation [11], recruiting

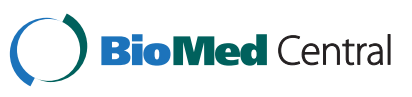


different muscle fiber types [12], synthesizing molecular chaperones [13], and altering mitochondrial densities and their properties [14]. Moreover, microarray techniques have been widely used to profile gene expressions in fishes exposed to short- or long-term cold stresses during the past decade. Researchers have characterized the transcriptional responses elicited by cold stress in fishes such as common carp (Cyprinus carpio) [15], zebrafish (Danio rerio) [16-18], channel catfish (Ictalurus punctatus) [19], annual killifish (Austrofundulus limnaeus) [20], coral reef fish (Pomacentrus moluccensis) [21] and rainbow trout (Oncorhynchus mykiss) [22]. These investigations have revealed a large number of cold-regulated genes involved in a variety of biological processes that are associated with acclimation to both daily and seasonal low temperatures.

It is well documented that the cold-tolerance of fishes is mainly determined by genetics, thermal history and developmental stages and fishes at larvae stages are more sensitive to cold injury than adults $[4,23,24]$. Despite the importance of larvae survival for fishery production and the stability of wild population, there is little information about the acclimation of fish larvae to cold stress. In some insects, a phenomenon named "rapid cold hardening $(\mathrm{RCH})$ " can be evoked by a mild cold exposure for a period of minutes or a few hours, which is important for protecting insects from cold injury at both organismal and cellular levels [25]. The $\mathrm{RCH}$ response has been described in both chill-sensitive and chill-tolerant insects and is remarkable for its rapid induction [26]. However, it is unknown whether fish larvae possess such a rapid acclimation response to cold stress and the transcriptional and posttranscriptional regulations in fish larvae exposed to cold stress remain to be defined.

Zebrafish is widely used as a research model for multiple disciplines including developmental biology, genetics, physiology, toxicology and environmental genomics. Abundant biological information and genetic resources have been accumulated for this species, e.g., the complete genome sequence and comprehensive annotations, which markedly facilitate investigations using high-throughput techniques such as microarray and RNA-seq. Moreover, zebrafish is a tropical eurythermal fish that might encounter a wide range of both daily and seasonal temperature fluctuations. Zebrafish acclimated to $20^{\circ} \mathrm{C}$ demonstrated critical thermal maxima (CTMax) and critical thermal minima (CTMin) of $39.2^{\circ} \mathrm{C}$ and $6.2^{\circ} \mathrm{C}$, respectively $[2,27]$. We have characterized the gene expression profiles in zebrafish larvae exposed to low or high temperature stress [18], but the post-transcriptional regulations remain unclear due to the limitations of microarray technique. RNASeq is a recently developed approach to determine the transcriptomic profiles using deep-sequencing technologies, which exhibits some advantages over microarray such as the independency of existing genomic sequence, high sensitivity and accuracy, digital expression and the ability to distinguish transcript isoforms [28].

This study aims to characterize the ability of zebrafish larvae to build cold-tolerance after exposed to a mild low temperature and investigate the transcriptional responses elicited by cold stress. We found that $96 \mathrm{hpf}$ zebrafish larvae exposed to a mild low temperature $\left(16^{\circ} \mathrm{C}\right)$ for $24 \mathrm{~h}$ exhibited a significant increase in survival rates under further severe cold stress at $12^{\circ} \mathrm{C}$. Transcriptional responses behind the formation of cold-tolerance were characterized using RNA-seq. The sequencing reads were mapped to zebrafish genome sequence and assembled into transcripts. A total of 23,693 genes were found to be expressed and differential expression analysis identified 1,431 up- and 399 down-regulated genes. Enrichment analysis of Gene Ontology (GO) terms and Kyoto Encyclopedia of Genes and Genomes (KEGG) pathways have revealed that RNA splicing, protein catabolic process and ribosome biogenesis were the most enriched GO terms and that spliceosome, proteosome and ribosome biogenesis in eukaryotes were the most overrepresented pathways among cold-induced genes. Furthermore, cold stress led to differential splicing of 197 genes and promoter switching of 64 genes. Obviously, these findings would be very important for further understanding the intracellular signaling mechanisms of cold stress in fish.

\section{Results}

\section{Development of cold resistance in zebrafish larvae}

To address whether zebrafish larvae are able to build cold-resistance under a mild low temperature, $96 \mathrm{hpf} \mathrm{lar-}$ vae were incubated at $16^{\circ} \mathrm{C}$ for $24 \mathrm{~h}$ followed by severe cold exposure at $12^{\circ} \mathrm{C}$ and the controls were maintained at $28^{\circ} \mathrm{C}$ before severe cold exposure at $12^{\circ} \mathrm{C}$ (Figure $1 \mathrm{~A}$ ). Larvae at $96 \mathrm{hpf}$ were used to perform the experiment because larvae at this stage need not to be fed and are less sensitive to cold stress than the earlier stage embryos [18]. As shown in Figure 1B, the death rates of the pre-treated larvae are significantly lower than those of the control at 12, 24, 36 and $48 \mathrm{~h}$ ( $\mathrm{p}<0.01$ in all cases). The death rates after severe cold exposure for 36 and $48 \mathrm{~h}$ reached $94.93 \%$ and $100 \%$ in the control, but were 35.71 and $55.93 \%$ in the pre-treated group. After severe cold exposure at $12^{\circ} \mathrm{C}$ for $36 \mathrm{~h}$, the survival larvae in experimental group were normal in morphology; however, most of the control larvae were dead and displayed an obvious body curvature (Figure 1C). These findings suggest that zebrafish larvae developed the resistance against severe cold stress after a pre-exposure at $16^{\circ} \mathrm{C}$.

\section{Mapping of RNA-seq reads to zebrafish genome}

To reveal the cold acclimation-associated transcriptional responses in larval zebrafish, three biological replicates of both control and cold-treated samples were subjected 


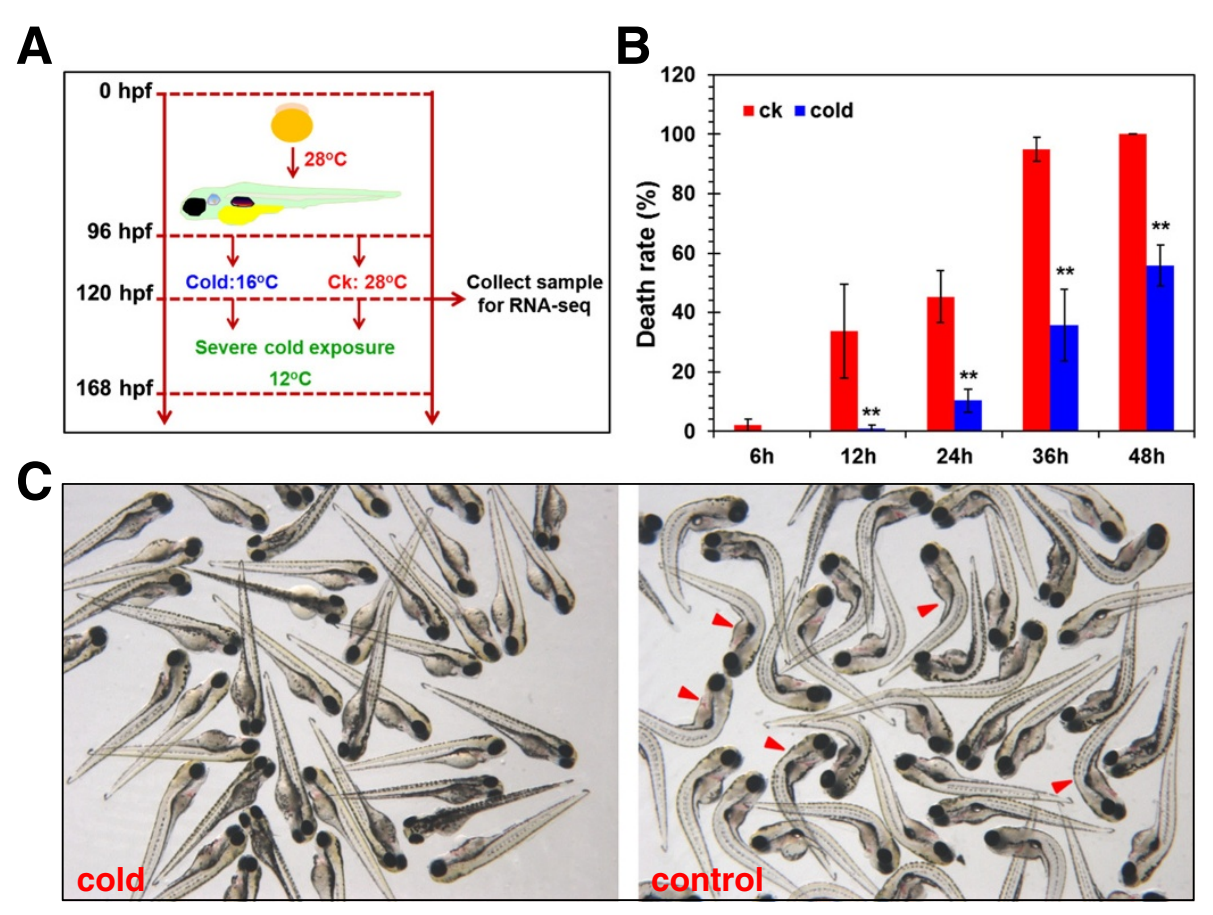

Figure 1 Establishment of cold resistance in zebrafish larvae after pre-exposure to cold stress. (A) Flowchart of cold exposure. Zebrafish embryos were incubated at $28^{\circ} \mathrm{C}$ from fertilization to $96 \mathrm{hpf}$. Larvae at $96 \mathrm{hpf}$ were exposed to $16^{\circ} \mathrm{C}$ for $24 \mathrm{~h}$ and the controls were maintained at $28^{\circ} \mathrm{C}$. Samples for RNA-seq were collected at $120 \mathrm{hpf}$. The pre-treated and control larvae were further exposed to $12^{\circ} \mathrm{C}$ for $6,12,24,36$ and $48 \mathrm{~h}$. The time scales were shown on the left. (B) Death rates of pre-treated and control (ck) larvae exposed to $12^{\circ} \mathrm{C}$ for different times. Data was shown as mean \pm standard deviation $(n=4)$. " $* * "$ above error bars indicate $p<0.01$. (C) Images of pre-treated and control larvae exposed to $12^{\circ} \mathrm{C}$ for $36 \mathrm{~h}$. Images were taken under a stereomicroscope from Zeiss with a color CCD camera. Red arrowheads indicate representative dead larvae.

to total RNA extraction and RNA-seq analysis. Highthroughput sequencing generated 19.48-26.51 million (M) pairs of raw reads for each of the sample and more than $96 \%$ of the raw reads passed quality filtering ( $\mathrm{Q}>20$ and length $\geq 25 \mathrm{bp}$ ) (Table 1). After the quality filtering, paired reads were extracted and mapped to the zebrafish genome using TopHat. A total of 37.18-50.20 M reads were processed by TopHat and the percent of mapped reads were quite similar among different samples (91.7792.56\%). The number of total mapping events generated by TopHat was 45.55-57.91 $\mathrm{M}$ and the number of potential splices was $5.27-5.32 \mathrm{M}$, representing $11.54-15.33 \%$ of the total alignment. The ratio of unique map to the total alignment was $75.25-78.50 \%$; however, the ratio of uniquely

Table 1 Statistics for the filtering and mapping of reads

\begin{tabular}{|c|c|c|c|c|c|c|}
\hline Sample name & ck1 & ck2 & ck3 & cold1 & cold2 & cold3 \\
\hline Total reads (M) & $19.80 \times 2$ & $24.72 \times 2$ & $19.48 \times 2$ & $26.51 \times 2$ & $21.07 \times 2$ & $24.57 \times 2$ \\
\hline Good reads (M) & 38.05 & 47.76 & 37.62 & 50.92 & 40.74 & 47.56 \\
\hline$\%$ Good reads & 96.09 & 96.61 & 96.58 & 96.06 & 96.67 & 96.80 \\
\hline Processed reads (M) & 37.52 & 47.22 & 37.18 & 50.20 & 40.25 & 46.95 \\
\hline Mapped reads (M) & 34.85 & 43. 40 & 34.38 & 46.06 & 37.26 & 43.34 \\
\hline \% Mapped & 92.89 & 91.90 & 92.47 & 91.77 & 92.56 & 92.33 \\
\hline Total alignment (M) & 45.55 & 56.44 & 45.57 & 57.91 & 47.28 & 55.32 \\
\hline Total potential splices (M) & 5.27 & 5.32 & 5.27 & 5.32 & 5.28 & 5.27 \\
\hline \% Reads mapped to junction & 15.13 & 12.26 & 15.33 & 11.54 & 14.16 & 12.16 \\
\hline Unique mapping (M) & 34.41 & 43.00 & 34.29 & 45.46 & 36.82 & 43.02 \\
\hline \% Unique mapping & 75.54 & 76.17 & 75.25 & 78.50 & 77.87 & 77.75 \\
\hline \% Uniquely mapped reads & 98.73 & 99.07 & 99.74 & 98.70 & 98.83 & 99.24 \\
\hline
\end{tabular}

$\%$ unique mapping indicates the ratio of unique mapping to the total alignment. 
mapped reads to the total number of processed reads was 98.73-99.74\% (Table 1).

\section{Gene expression detected by RNA-seq}

The mapping data generated by TopHat was processed by Cufflinks toolkits for transcript assembly and differential expression analysis. The abundance of gene transcripts was expressed as FPKM (Fragments per kilobase of transcript per million fragments mapped) [29]. To identify expressed genes, background coverage for intergenic regions was calculated as well. As shown in Additional file 1, the mean background coverage was 0.029 FPKM and a significant decrease in frequency occurred at 0.1 FPKM. Therefore, genes with a mean abundance $>0.1$ FPKM in either the control or cold-treated samples were regarded as being expressed and a total of 23,693 expressed genes were detected in this study (Table 2).

As shown in Figure 2A and B, the abundance of most genes was less than 100 FPKM and both the control and cold-treated samples demonstrated a bimodal frequency curve of FPKM values. The first peak of the frequency curves was located at 0.1 FPKM and the second peak was located at 5.38 and 6.45 FPKM for the control and cold-treated samples, respectively. The trough between the two peaks was near 1 FPKM and the baselines of the second frequency peak range from 1 to 20 FPKM for both groups (Figure $2 \mathrm{~A}$ and $\mathrm{B}$ ). Therefore, genes were classified as low $($ FPKM $\leq 1)$, medium $(1<$ FPKM $\leq 20)$ and high abundance (FPKM $>20)$ according to their FPKM values. The largest portion (more than $60 \%$ ) of expressed genes belongs to the medium-abundance, followed by lowabundance genes (nearly $23 \%$, Additional file 2). A smaller number of medium-abundance genes and a larger number of high-abundance genes were detected in cold-treated samples than those in the control, suggesting that the expression of many genes was induced in cold-treated larvae.

Principle component analysis (PCA) was performed to characterize the overall effects of cold stress on gene expression. The results of PCA indicate that $78.52 \%$ of the variations in gene expression can be explained by the first two principle components $(\mathrm{PC})$. The inter-group and intra-group variations in gene expression were captured by $\mathrm{PC} 1$ and PC2 respectively (Figure 2C). PCA projections of the control and cold-treated samples in

\section{Table 2 Statistics of genes regulated by cold stress}

\begin{tabular}{lc}
\hline Classification & Number \\
\hline Detected genes & 23693 \\
Up-regulated genes & 1431 \\
Down-regulated genes & 399 \\
Differentially spliced genes & 197 \\
Genes with promoter-switching & 64 \\
\hline
\end{tabular}

the principle component space exhibited a clear discrepancy, indicating that significant variations in gene expression occurred after cold exposure.

The differentially expressed genes between the control and cold-treated groups were displayed in Figure 2D. Genes with fold change $\geq 2$, p-value $<0.05$ and q-value $<0.05$ were considered to be differentially expressed. The numbers of up- and down-regulated genes after cold exposure were 1,431 and 399 (Table 2). Genes regulated by cold stress were listed in Additional file 3. Genes such as il12a (interleukin 12a) and $s p 8 b$ (sp8 transcription factor b) were found to be specifically expressed in cold-treated larvae. Genes ptgr1 (prostaglandin reductase 1), irg1l (immunoresponsive gene 1, like) and mmp13a (matrix metalloproteinase 13a) were among the most highly up-regulated genes under cold stress, while mep1b (meprin A, beta), rh50 (Rh50like protein) and $n r 5 a 5$ (nuclear receptor subfamily 5 , group A, member 5) were representative genes inhibited by cold stress. A comparison with our previous study [18] revealed a significant overlap between the up-regulated genes detected by microarray and RNA-seq (Additional file 4). Since larvae exposed to cold stress for different time periods were used in these studies, considerable discrepancy in gene expression was also found.

\section{Validation of RNA-seq data by quantitative real time PCR (qPCR)}

To validate the expression profiles from RNA-seq analysis, relative mRNA levels for 15 genes were measured by qPCR. The expression data for these genes and transcript isoforms detected by RNA-seq and qPCR are displayed in Table 3 and Figure 3. Expression of these genes and transcripts except cry-dash, were confirmed by qPCR. The downregulation of per3 upon cold stress was detected by probes targeting per3-J1 and per3-J2 (transcripts of per3-P1 in Table 3 and Figure 4) in our previous microarray study [18]. However, RNA-seq explicitly revealed the downregulation of per3-J1 and per3-J2 and the up-regulation of per3-J3 (transcript of per3-P2 in Table 3 and Figure 4) in this study. The data from qPCR and RNA-seq exhibited excellent agreement on both up- and down-regulated genes. The correlation between microarray and qPCR data was analyzed by Spearman's rho test and a highly statistical significance $[\mathrm{r}(17)=0.938, \mathrm{p}=0.000001]$ was observed.

\section{Gene ontology (GO) enrichment analysis of genes regulated by cold stress}

GO enrichment analysis was performed to reveal the biological processes overrepresented under cold stress. As shown in Figure 5, five main functional categories including RNA metabolic process, cellular component biogenesis, regulation of metabolic process, catabolic process and RNA localization were significantly enriched from genes up-regulated by cold stress. More specific terms of 

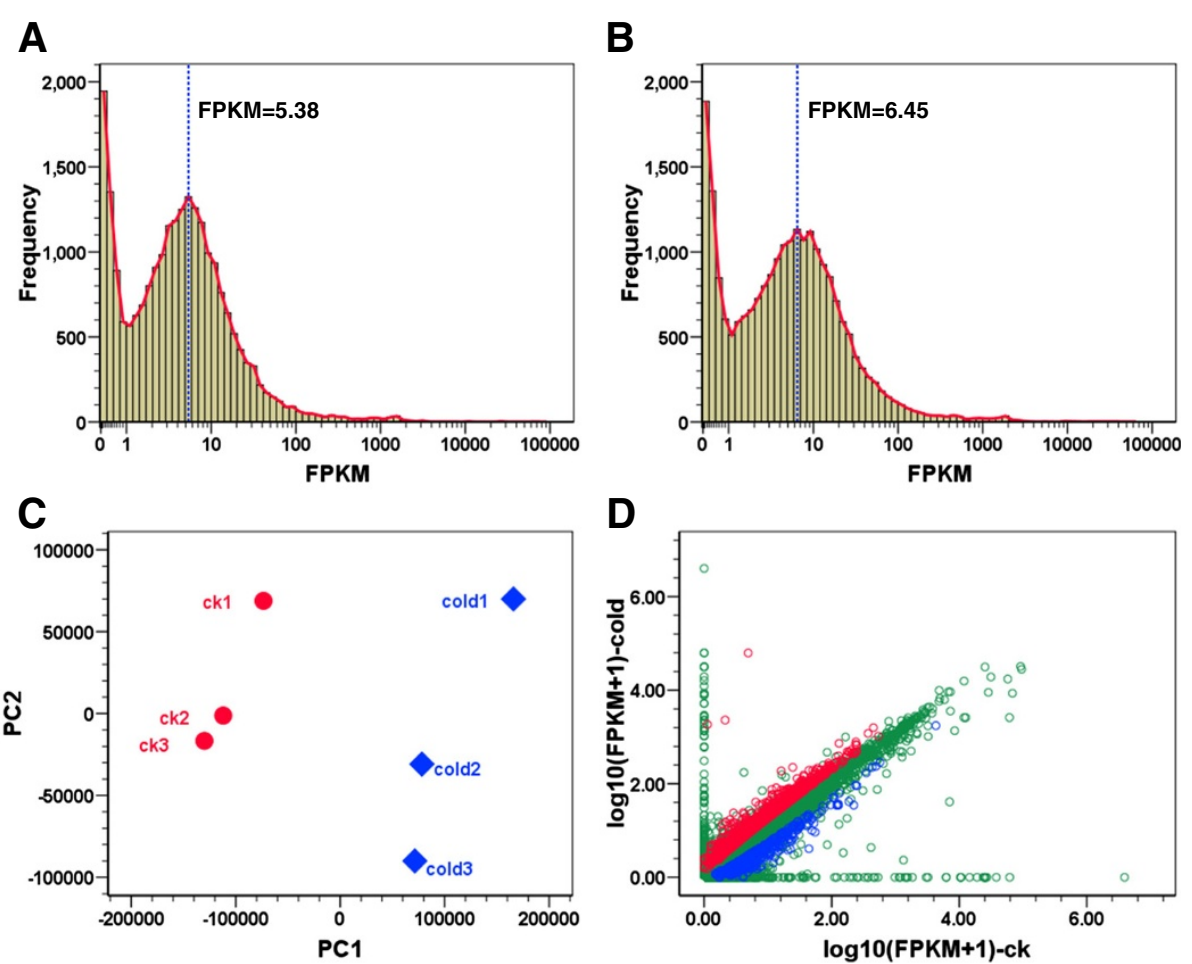

Figure 2 Bioinformatic analysis of RNA-seq data. (A and B) Distribution of FPKM values for genes expressed in the control (A) and cold-treated (B) zebrafish larvae. The red interpolation line denotes a bimodal distribution of the frequency of FPKM. The blue dashed line indicates the FPKM value for the second peak of frequency. (C) Principle component analysis of gene expression in the control (ck) and cold-treated samples. (D) Correlation of gene expression between the control and cold-treated group. The up- and down-regulated genes were shown in red and blue, respectively. Genes not regulated by cold treatment were shown in green.

Table 3 Comparisons between RNA-seq data and qPCR results

\begin{tabular}{|c|c|c|c|}
\hline \multirow[t]{2}{*}{ Gene symbol } & \multirow[t]{2}{*}{ Gene name } & \multicolumn{2}{|c|}{ Fold change } \\
\hline & & qPCR & RNA-seq \\
\hline brf2 & BRF2, subunit of RNA polymerase III transcription initiation factor, BRF1-like & 3.3 & 5.9 \\
\hline per2 & Period homolog 2 & 3.0 & 5.9 \\
\hline$n r f 1$ & Nuclear respiratory factor 1 & 2.3 & 2.5 \\
\hline fosl1a & FOS-like antigen 1a & 2.1 & 5.1 \\
\hline gabpa & GA-binding protein transcription factor, alpha subunit & 2.0 & 2.8 \\
\hline per1b & Period homolog $1 b$ & 2.0 & 4.4 \\
\hline Per3-total & Period homolog 3 & 2.9 & 5.8 \\
\hline per3-P1 & Period homolog 3 & -4.8 & -3.0 \\
\hline per3-P2 & Period homolog 3 & 10.0 & 56.3 \\
\hline fos & v-fos FBJ murine osteosarcoma viral oncogene homolog & 2.5 & 3.1 \\
\hline stk16-j1 & Serine/threonine kinase 16 & 1.4 & 1.5 \\
\hline cry-dash & Cryptochrome DASH & 1.3 & 2.3 \\
\hline her8a & Hairy-related 8a & -2.5 & -2.1 \\
\hline setd7 & SET domain containing (lysine methyltransferase) 7 & -2.7 & -2.5 \\
\hline sirt3 & Sirtuin (silent mating type information regulation 2 homolog) 3 & -2.7 & -2.7 \\
\hline stc1l & Stanniocalcin 1, like & -2.8 & -2.1 \\
\hline$n r 5 a 5$ & Nuclear receptor subfamily 5 , group A, member 5 & -5.7 & -9.4 \\
\hline
\end{tabular}




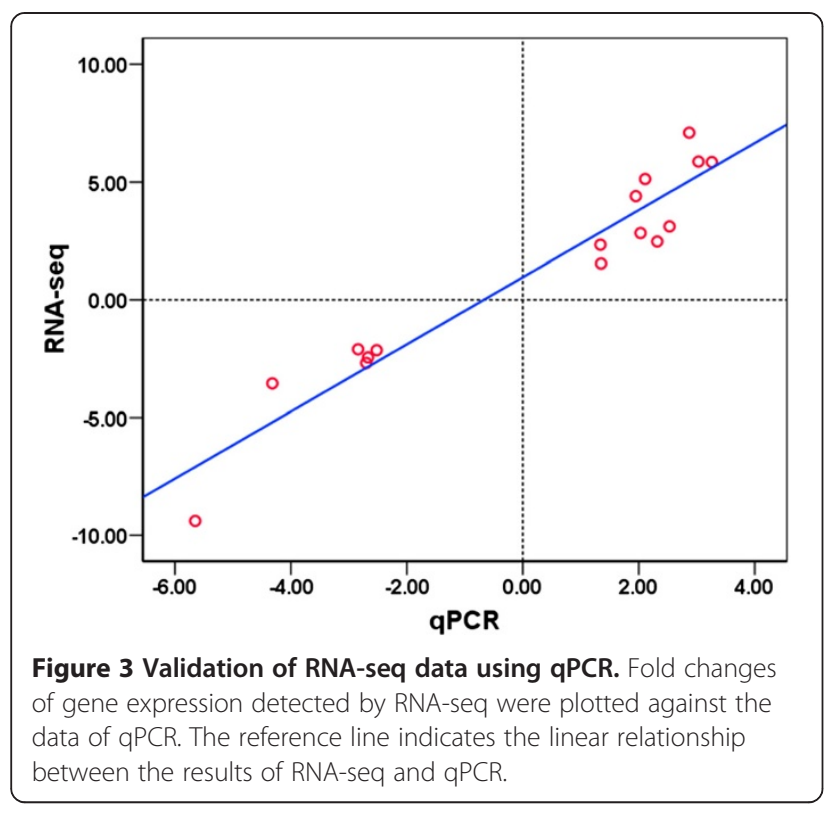

these enriched categories include RNA splicing, ribosome biogenesis and protein catabolic process (Figure 5).

The most specific GO terms overrepresented by coldinhibited genes include oxidation-reduction process, fatty acid biosynthetic process, proteolysis, sterol biosynthetic process, ammonium transmembrane transport, oligopeptide transport, organic cation transport and chitin catabolic process (Figure 6). Genes associated with the enriched GO terms were shown in Additional file 5. Some of these enriched GO terms from this study such as RNA processing, ubiquitin-dependent protein catabolic process and proteolysis were previously found to be overrepresented in coldregulated genes [15,18]. The results of GO enrichment analysis indicate that multiple biological processes are involved in the establishment of cold resistance in zebrafish larvae.

\section{KEGG pathway enrichment analysis of genes regulated by cold stress}

The results of KEGG pathway enrichment analysis were displayed in Additional file 6. A total of 61 pathways were overrepresented in genes up-regulated by cold stress. Spliceosome was the most significantly enriched pathway, in which $43.33 \%$ (52) of the associated genes were upregulated by cold stress. The components of spliceosome include the U1, U2, U4, U5 and U6 small nuclear ribonucleoprotein particles (snRNPs), each of which consists of a specific small nuclear RNA (snRNA), a common set of seven $\mathrm{Sm} / \mathrm{Lsm}$ proteins and variable number of particlespecific proteins [30,31]. We found that the Sm/Lsm genes and nearly all others associated with $\mathrm{U} 2, \mathrm{U} 4 / \mathrm{U} 6$ and

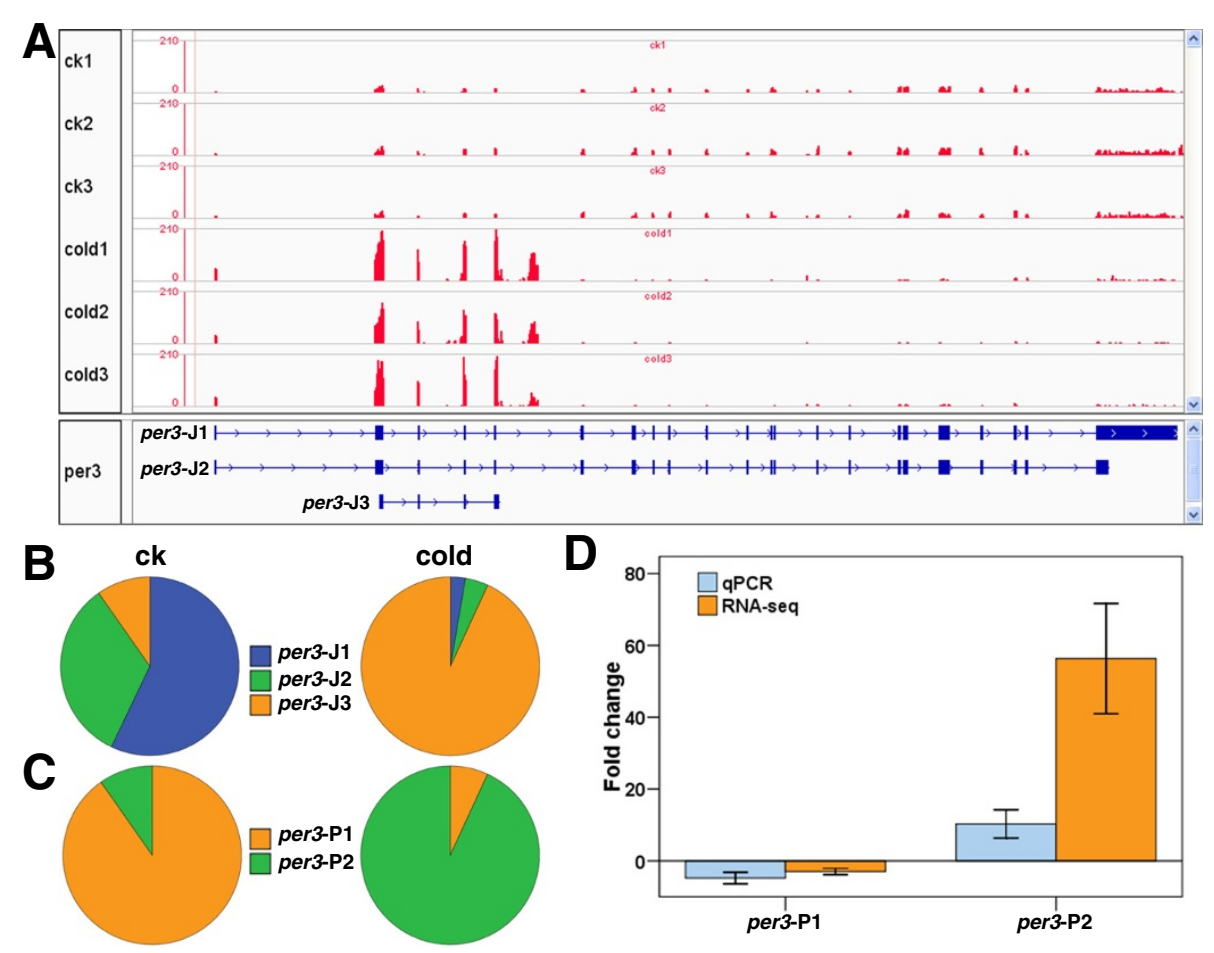

Figure 4 Alternative promoter usage of per3 under cold stress. (A) Read coverage at per3 locus. The top panel shows the read coverage of each sample at per3 locus and the bottom panel indicates the structure of per3 transcripts. (B) The relative abundance of per3 isoforms under cold stress. (C) Promoter switching of per3 under cold stress. (D) qPCR validation of the alternative promoter usage for per3. Error bars indicate standard deviation $(n=3)$. 

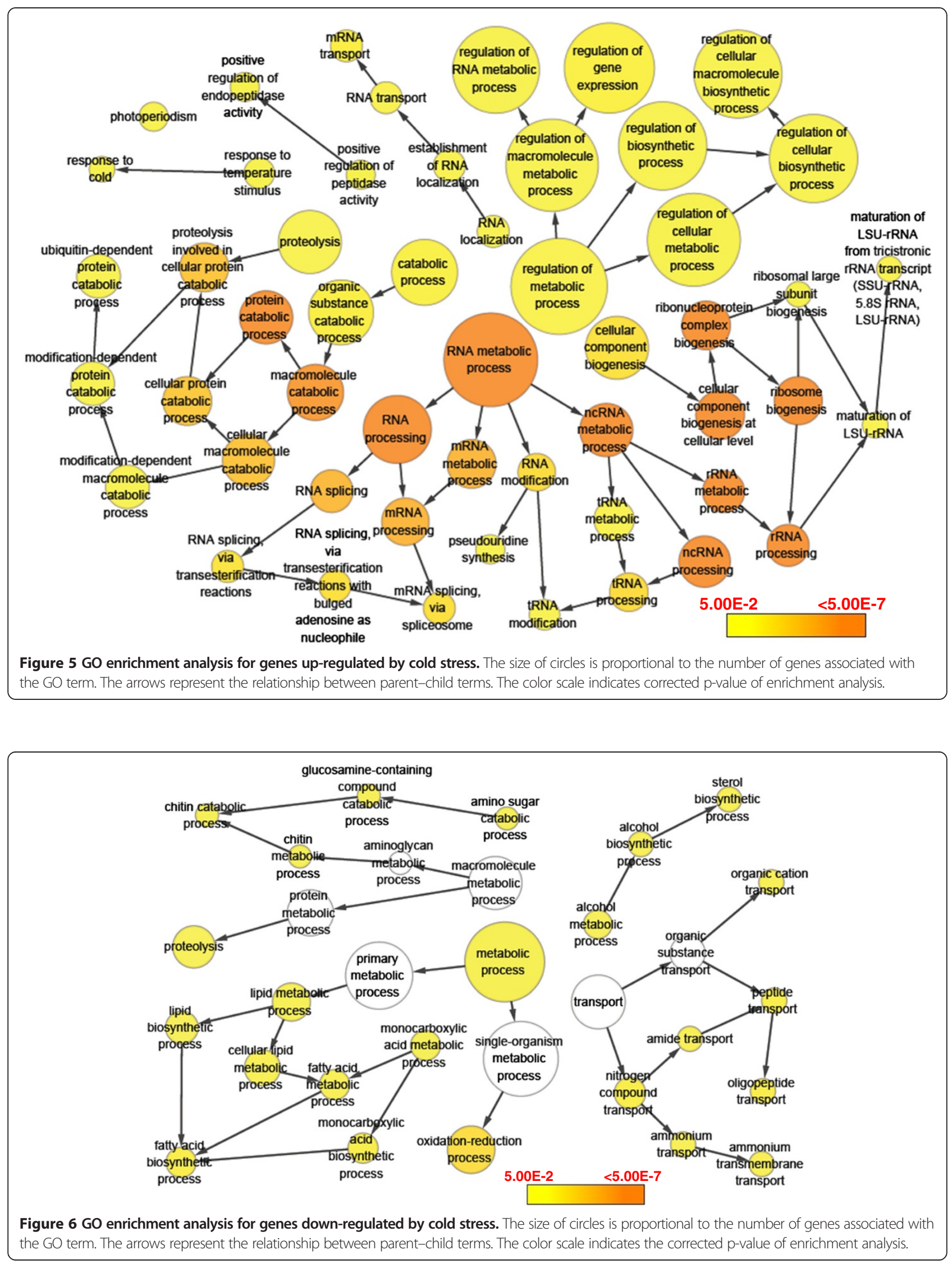
U5 were up-regulated by cold stress (Figure 7). Some of the cold-induced genes have been shown to play essential roles in splicing. For example, U2 specific splicing factors SF3a (splicing factor 3a, subunit 2) and SF3b (splicing factor 3B subunit 3-like) are involved in stabilizing the U2/ BS (Branch site) duplex [31], and members of the DExD/ H-box family of RNA unwindases/RNPases such as Brr2 (pre-mRNA-splicing helicase BRR2), Prp28 (DEAD box polypeptide 23) and Prp43 (DEAH box polypeptide 15) are responsible for driving rearrangements of the spliceosome's RNA-RNA and RNA-protein networks required for splicing [31,32]. In addition, some genes involved in the nonsnRNPs complexes such as Prp19 complex were upregulated by cold stress as well (Figure 7).

Ribosome biogenesis in eukaryotes is another highly represented pathway with $30.88 \%$ (21) of the associated genes up-regulated by cold stress (Additional file 6 and Figure 8). The process of ribosome biogenesis involves the maturation of ribosomal rRNAs and their assembly into ribosomal subunits [33]. Among the multiple steps during the ribosomal subunit biogenesis, cold-induced genes mainly function in the rRNA modification process. The 2'-O-methylation and pseudouridylation are the most prevalent modifications of rRNAs. The modified nucleotides are important for the conformation and stabilization of RNA and the activity of ribosome in translation [33]. Among the cold-induced genes associated with ribosome biogenesis, Nop1 (fibrillarin), Nop56 (NOP56 ribonucleoprotein homolog), Nop58 (NOP58 ribonucleoprotein homolog, yeast) and Snu13 (NHP2 non-histone chromosome protein 2 -like $1 \mathrm{~b}$, yeast) are associated with $2^{\prime}$-Omethylation. Dkc1 (dyskeratosis congenita 1, dyskerin), Nhp2 (NHP2 ribonucleoprotein homolog, yeast) and Gar1 (GAR1 ribonucleoprotein homolog, yeast) are related to pseudouridylation of rRNAs. Utp5 (WD repeat domain 43), Utp10 (HEAT repeat-containing protein 1-like), Utp15 (U3 small nucleolar ribonucleoprotein, homolog) and Nan1 (WD repeat domain 75) are building blocks of the

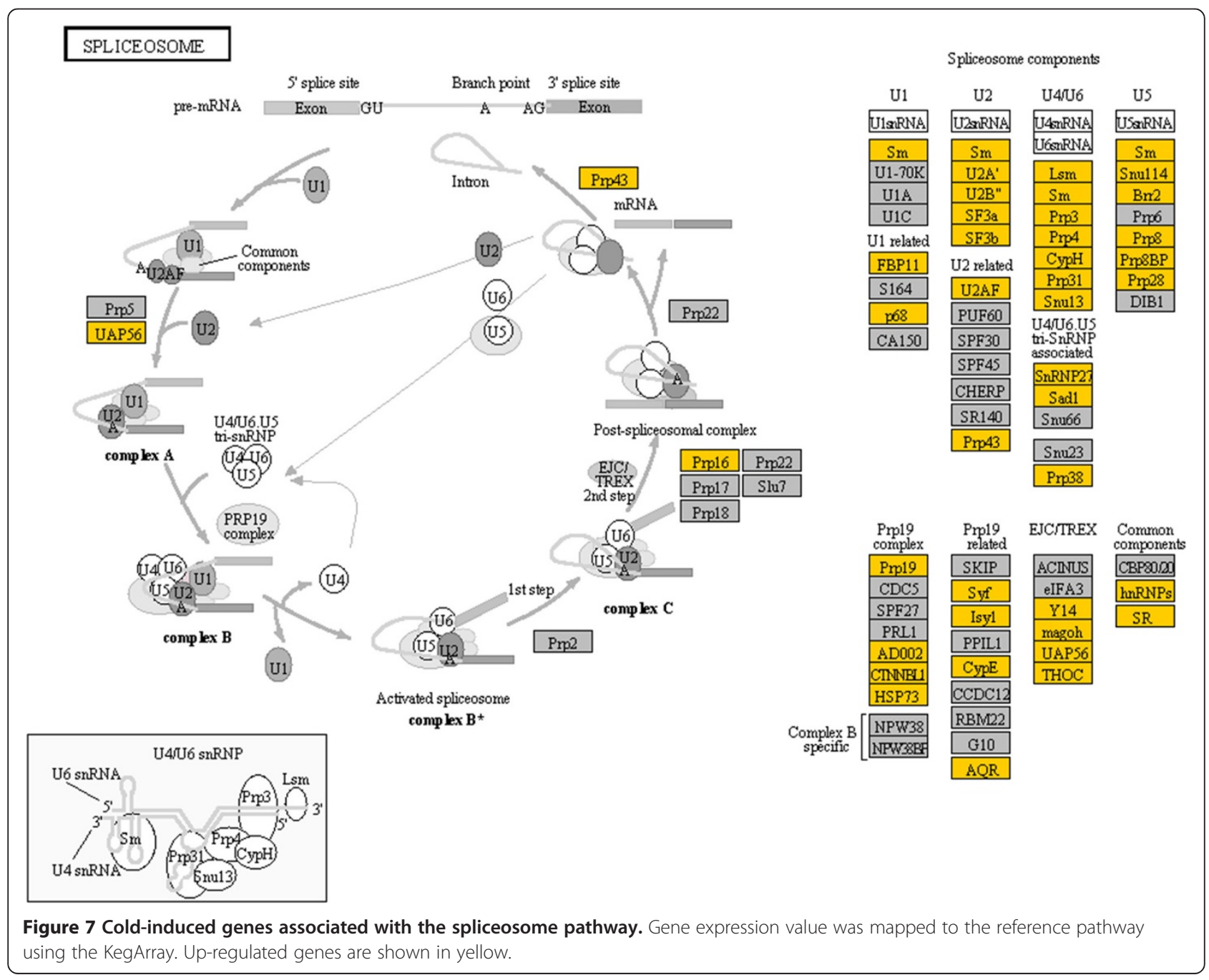




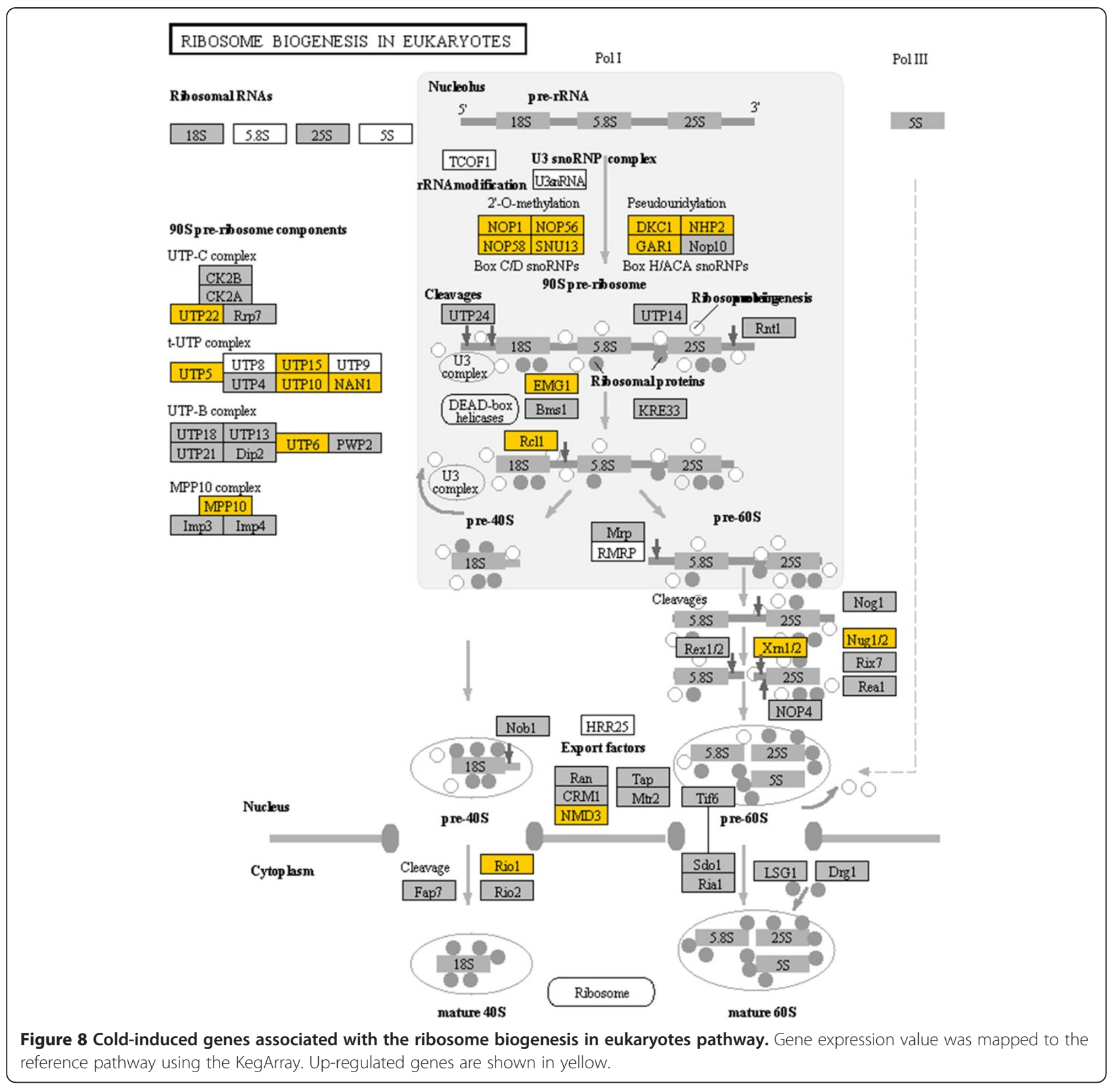

t-UTP complex, which is required for the subsequent assembly of other 90S pre-ribosome components [34]. Additionally, genes associated with other $90 \mathrm{~S}$ pre-ribosome components and the maturation of $40 \mathrm{~S}$ and $60 \mathrm{~S}$ subunits were up-regulated by cold stress (Figure 8).

Other representative pathways regulated by cold stress include RNA transport, pyrimidine metabolism, RNA polymerase, purine metabolism, mRNA surveillance pathway, herpes simplex infection, protein processing in endoplasmic reticulum and circadian rhythm. Moreover, many pathways involved in signal transduction such as MAPK signaling, p53 signaling, ErbB signaling, Wnt signaling and mTOR signaling pathways were enriched from cold- induced genes. Furthermore, pathways associated with cell communication such as tight junction and gap junction were overrepresented in cold-induced genes. These findings indicate the importance of signal transduction and cell communication in the acclimation of zebrafish larvae to cold stress.

Pathways overrepresented by cold-inhibited genes include steroid biosynthesis, peroxisome, drug metabolism, amino sugar and nucleotide sugar metabolism, inositol phosphate metabolism, cardiac muscle contraction (Additional file 6). Since most of these pathways are associated with metabolism, cold stress appears to suppress the basal metabolism of zebrafish larvae. 


\section{Differential splicing under cold stress}

In addition to differentially expressed genes, Cuffdiff can identify genes with differential splicing and promoter switching under different conditions [35]. A total of 197 genes were found to be differentially spliced in zebrafish larvae after exposure to cold stress (Additional file 7). Results of functional clustering reveal that these differentially spliced genes are classified into eight functional clusters in which regulation of transcription is the most highly represented biological process (Additional file 8). The expression of stk16 (Ser/Thr kinase 16) was characterized as an example of posttranscriptional regulation induced by cold stress. Zebrafish stk16 gene is located on chromosome 1, which can generate one transcript as predicted in Ensembl (http://www.ensembl.org/). This transcript encodes a peptide of 306 amino acids (designated as stk16-J1). Cufflincks assembled a new transcript (stk16-J2) lacking the second exon in stk16-J1 and the coverage of all other exons was markedly increased in coldtreated samples when compared to the controls (Figure 9A). The results of differential expression analysis revealed that stk16-J2 was specifically expressed in larvae exposed to cold stress (Figure 9B). RT-PCR analysis confirmed the specificity of stk16-J2 in cold-treated samples (Figure 9C). The skipping of exon 2 led to a truncated peptide lacking the $\mathrm{N}$-terminal 67 amino acids (Figure 9D).

\section{Alternative promoter usage under cold stress}

When a gene is transcribed from multiple transcription start sites (TSSs) due to the alternative usage of promoters, Cuffdiff will allocate them into different TSS groups by adding up the expression levels of isoforms, which reflect the promoter preference between conditions [35]. In this study, putative promoter switching was found in 64 genes under cold stress (Additional file 9). Among these genes, zebrafish per3 gave rise to three transcripts including per3$\mathrm{J} 1$, per3-J2 and per3-J3 (Figure 4A). Per3-J1 and per3-J2 share the same TSS (per3-P1) and open reading frame, while per3-J3 uses a different TSS (per3-P2) to generate a short transcript (Figure 4A). The expression level of per3-J3 was significantly induced, but those of per3-J1 and per3-J2 were inhibited by cold stress (Figure 4A and B). Moreover, the per3-J3 became the dominant transcript under cold stress, indicating per3-P2 is a cold- preferred promoter (Figure 4C). The up-regulation of per3-P2 under cold stress was further validated by qPCR analysis (Figure 4D).

Since zebrafish per 3 was reported to be a circadian gene [36], we further characterized the expression pattern of per3 transcripts during a $24 \mathrm{~h}$ light-dark cycle. Zebrafish larvae were kept under light-dark cycle from fertilization and exposed to $16^{\circ} \mathrm{C}$ from $96 \mathrm{hpf}$ to $144 \mathrm{hpf}$. As shown in Additional file 10, the overall expression of per3-P1 transcripts under $28^{\circ} \mathrm{C}$ peaked at 12:00 am and

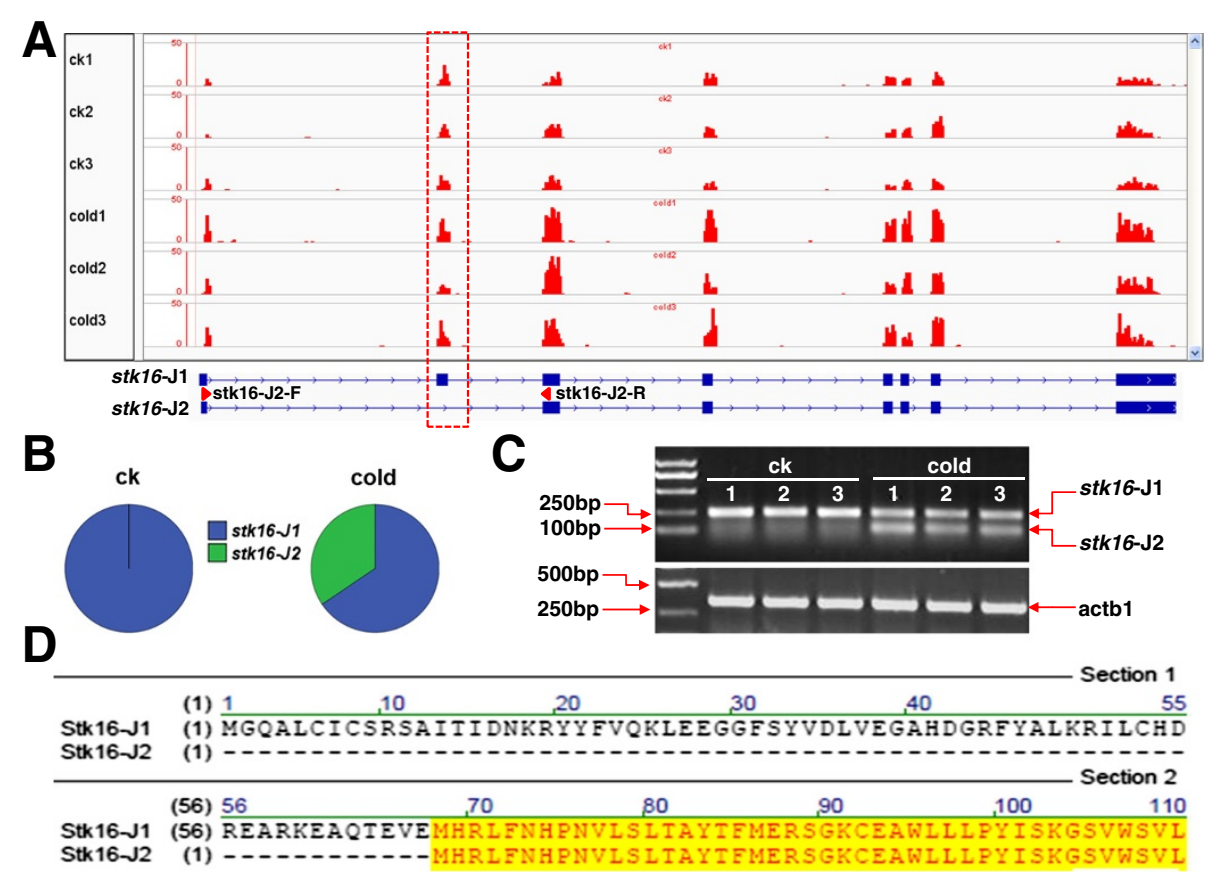

Figure 9 Differential splicing of stk16 under cold stress. (A) Read coverage at stk16 locus. The top panel shows the read coverage of each sample at sk16 locus and the bottom panel indicates the structure of stk 16 transcripts. The red arrowheads represent relative positions of primers for RT-PCR. The dashed red box indicates the unchanged coverage of exon 2 under cold stress. (B) RT-PCR analysis to reveal the cold-specificity of stk16-J2. Stk16-J2-F and stk16-J2-R primers were displayed in (A). (C) The relative abundance of stk16 isoforms under cold stress. (D) Partial peptide sequence alignment of Stk16-J1 and Stk16-J2. The identical amino acids are shown in yellow. 
dropped to trough at 12:00 pm; however, when exposed to cold stress the level of per3-P1 transcripts decreased at first and peaked at 8:00 pm. The level of per3-J3 was very low and no diurnal expression patterns were found in control larvae maintained at $28^{\circ} \mathrm{C}$. Upon cold exposure, the expression level of per3-J3 continuously increased and the peak was found at 8:00 pm (Additional file 10). These results indicate that cold stress can lead to the alteration in the circadian rhythm of longer per3 transcripts per3-J1 and per3-J2, and induce the shorter transcript per3-J3 showing no diurnal expression patterns under normal temperature.

\section{Discussion}

It has been suggested that genetic background, thermal history and developmental stages are the most important factors determining the cold-tolerance of fish $[2,4,24]$. Most of the previous studies of cold acclimation in fish have focused on long-term and especially seasonal adaptive responses in juveniles or adults. It was recently shown that the thermal experience of fish at embryonic stages can have dramatic and persistent effects on thermal acclimation capacity of adults [37,38]. However, the adaptive responses of fish larvae to cold stress and the transcriptional alterations during these processes remain largely unknown. In this study, we demonstrated that exposure of $96 \mathrm{hpf}$ zebrafish larvae to $16^{\circ} \mathrm{C}$ for $24 \mathrm{~h}$ led to a significant increase in survival rates of developing larvae under further severe cold stress at $12^{\circ} \mathrm{C}$, indicating that zebrafish larvae possess a $\mathrm{RCH}$-like ability to build cold-tolerance under mild low temperature. To our knowledge, this is the first evidence indicating the existence of $\mathrm{RCH}$-like responses in fish. In insects, the elevated body levels of low molecular cryoprotectants such as glucose, trehalose and glycerol were suggested to be the biochemical basis of the $\mathrm{RCH}[26,39]$. In fishes, increases in the ratio of unsaturated fatty acids and plasma lactate concentration [7], upregulated mitochondria biogenesis [10], biosynthesis of isoenzymes [9] and regulation of muscle fiber types [40] were found to be associated with long-term cold acclimation. To further address the molecular mechanisms underlying the establishment of cold-tolerance in fish larvae, we investigated the transcriptional responses during the establishment of cold-tolerance using RNA-seq. A total of 1431 up- and 399 down-regulated genes were identified in 23693 expressed genes of zebrafish larvae. These differentially expressed genes are involved in many crucial processes and pathways such as RNA splicing, protein catabolic process, ribosome biogenesis, spliceosome, proteosome and ribosome biogenesis. Additionally, the alternative splicing of 197 genes and promoter switching of 64 genes were found to be specifically regulated by cold stress. Obviously, findings of the study are of great importance for further investigation of the cold-specific signaling networks in fish.
Previous studies have shown that a very large number of genes are involved in the acclimation of fish to cold stress. A systematic microarray study of carp tissues exposed to low temperature for 22 days identified 3461 cold-regulated cDNAs [15]. Many of cold-induced genes were found to be regulated by both short- and long-term cold stress. Representatives of these genes include coldinducible RNA binding protein (cirbp), high-mobility group proteins ( $h m g b 1 a, h m g b 1 b)$, ribonucleoproteins involved in mRNA processing and splicing (snrpd3, snrpa1, prpf8 and sf $3 a 2$ ) and translation initiation factors (eiflaxa, eiflaxb, eief $2 a$ and denr). Thus, these genes can be used as potential molecular markers for characterization of the cold acclimation responses in different fish species. Moreover, many of the highly overrepresented functional categories and pathways of cold-induced genes from this study are overlapped with those found in all the analyzed tissues of carp exposed to cold stress [15], indicating the importance of these processes or pathways in the establishment of cold-tolerance. Since RNA processing and ribosome biogenesis processes are directly associated with gene expression, the alterations of these biological processes are consistent with the notion that more up-regulated genes are usually found than downregulated genes in transcriptomic analysis of fishes exposed to cold stress $[15,19]$. Furthermore, protein catabolic processes such as the ubiquitin-dependent protein catabolism were often found to be induced by cold exposure, suggesting the degradation and modification of unfolded or misfolded proteins under low temperature stress.

The sensing and intracellular transduction of stress signals is critical for the adaptation and survival of organisms under various environmental stresses. Information about the mechanisms whereby fish cells sense the cold signal and trigger the intracellular responses is extremely scarce. In this study, mitogen-activated protein kinase (MAPK), p53 and peroxisome proliferator-activated receptor (PPAR) signaling were found to be the most highly enriched signal transduction pathways under cold stress. The canonical MAPK signaling pathway is a threecomponent signal cascade in which an activated MAPK kinase kinase (MAPKKK or MEKK) activates a MAPK kinase (MAPKK or MEK), which then activates an extracellular signal-regulated kinase MAPK or ERK [41]. It has been shown that MAPK pathway is involved in intracellular responses to diverse environmental stresses including cold, heat, reactive oxygen species, UV, desiccation and pathogen attack $[42,43]$. The p53 signaling pathway can be induced by stress signals such as DNA damage, hypoxia, heat and oxidative stress and its activation often leads to cell cycle arrest, cellular senescence or apoptosis [44-46]. The PPAR signaling pathway is mainly involved in the protection of cells against oxidative stress and apoptosis $[47,48]$. Overrepresentation of these signaling pathways among cold-induced genes suggests their importance in 
the transduction of cold signals and the establishment of cold-tolerance in fish. Since protein phosphorylation is extensively involved in the activation of these signaling pathways, characterizing the phosphorylation of intracellular proteins upon cold stress would provide further insights into the networks for sensing and transduction of cold signal.

Alternative splicing events such as exon skipping, alternative usage of $5^{\prime} / 3^{\prime}$ splice sites and intron retention may lead to changes of the amino- or carboxy-terminus, in-frame addition/removal of a functional unit and insertion of premature termination codon, thus contributing to both transcriptomic and proteomic diversities [49]. Accumulating evidence indicates that alternative splicing is important for adaptation responses to a wide range of stress conditions [50-54]. Stress-associated alternative splicing is mainly described for genes encoding the protein kinases, transcription factors, splicing regulators and pathogen-resistance factors, and the alternative splicing under stress conditions usually leads to changes in the subcellular localization, binding properties, and activity or stability of the resulting proteins [49]. In this study, alternative splicing of 197 genes and promoter switching of 64 genes were found to be regulated by cold stress. The generation of a short stk16 splicing isoform under cold stress was selected for validation of transcriptome analysis. It is known that STK16 functions as a transcriptional coactivator in the expression regulation of vascular endothelial growth factor (VEGF) [55]. However, the functional significance of alternative splicing of zebrafish stk16 under cold stress remains unclear. Moreover, promoter switching was confirmed by molecular analysis of per3 transcripts and a short isoform containing 4 exons was found to be highly up-regulated under cold stress. It has been demonstrated that per3 is involved in multiple processes such as circadian rhythm, sleep, cancer, cell proliferation and apotosis [56-58]. Further investigations are needed to reveal the functions of cold-specific splicing variants and transcripts in the cold acclimation of zebrafish.

Nearly all eukaryotic cells possess self-sustained circadian clocks that couple endogenous biochemical, physiological and behavioral rhythms with environmental changes [59]. The effects of temperature on circadian clocks have been well established. Except for day/night cycles, temperature changes serve as a zeitgeber for circadian clocks and temperature cycles of as little as $2^{\circ} \mathrm{C}$ are sufficient to entrain the clock [60-62]. Temperature changes in physiological range can influence the amplitude of circadian transcriptional rhythms but not alter the period length of circadian cycles (temperature compensation) [61,62]. Out of the range of temperature compensation, the clock stops running and arrests at a certain phase [63]. In plants, coldresponsive pathways and cold tolerance are intimately associated with circadian clocks [64-66]. In zebrafish, many core clock genes such as nuclear receptors (nr1d1, nr1d2a, nr1d2b, nr1d4a and nr1d4b), period homologs (per1a, per1b, per2 and per3) and cryptochromes (cry1a, cry1b, cry2 and cry $2 b$ ) were induced by cold stress (Additional file 3), suggesting the involvement of circadian clocks in cold acclimation. Furthermore, we identified a shorter isoform of per3 gene highly up-regulated by cold stress (Additional file 10). This short isoform lacks the functional PAS (Per-Arnt-Sim) domain and the period circadian-like C-terminal domain and is similar to a small interference peptide (siPEP), which serves as a dominantnegative to interfere the activities of corresponding transcription factors in plants under cold stress [67]. Further investigations are needed to address if the cold-specific transcription of per 3 is related to temperature compensation of circadian clock and cold acclimation in zebrafish.

Cells adapt to stresses or changing environmental conditions as a result of alterations in gene expression at multiple levels including transcriptional, post-transcriptional and translational regulations [68]. Although a large number of genes were found to be up-regulated at transcriptional level upon cold stress, the correlation between gene transcription and the proteomic landscape remains to be characterized. The activity of proteins is dependent on their correct folding and only correctly folded proteins have the long-term stability in crowded biological environments and are able to interact selectively with their natural partners [69]. The folding of proteins is a temperaturedependent process and the hydrophobic effect driving protein folding decreases with dropping temperature [70,71]; therefore, the folding rate of proteins decreases at reduced temperature [72]. The landscape of protein folding in living cells can affect gene expression at transcriptional level, so the up-regulated mRNA level of some genes and alternative splicing events under cold stress could be a compensating measure for the reduced protein folding rate. Further investigations are needed to address the correlation between cold stress-induced transcriptomic and proteomic responses in fish.

\section{Conclusions}

This study has revealed the existence of cold acclimation in zebrafish larvae. Further transcriptomic analysis has uncovered many cold-regulated genes encoding proteins that are key components of some crucial biological processes and signaling pathways such as RNA splicing, ribosome eukaryotic ribosome biogenesis, protein catabolism, spliceosome, proteasome and RNA transport. Additionally, a large number of alternative splicing and promoter switching events were identified to be specifically regulated during the establishment of cold acclimation in zebrafish. These findings have provided novel clues for further investigation of the molecular mechanisms underlying the cold acclimation in zebrafish. 


\section{Methods}

\section{Animals and cold exposure}

The animal protocol for this study was approved by the Institutional Animal Care and Use Committee of Institute of Hydrobiology (Approval ID: Y21304501). Maintenance of adult zebrafish and incubation of embryos were performed as previously described [18,73]. Biochemical incubators (HWS-150, Shanghai Jinghong laboratory instrument Co., Ltd.) were used for temperature control and incubation of embryos. During cold exposure, zebrafish larvae at $96 \mathrm{hpf}$ were transferred immediately into dishes (50 larvae per dish) containing culture medium preconditioned at $16^{\circ} \mathrm{C}$ and incubated for $24 \mathrm{~h}$ (Figure 1). The controls were maintained at $28^{\circ} \mathrm{C}$. No embryonic mortality was observed during the treatment. After cold exposure, the dishes were placed on ice for $5 \mathrm{~min}$ to anesthetize the larvae and samples were collected and subjected to RNA extraction for preparation of RNA library followed by RNA-seq.

To evaluate the ability of zebrafish larvae to build cold resistance after pre-exposure to $16^{\circ} \mathrm{C}$, pre-treated and control larvae at $120 \mathrm{hpf}$ were further exposed to severe cold stress at $12^{\circ} \mathrm{C}$ for $6,12,24,36$ and $48 \mathrm{~h}$, respectively (Figure 1A). The larvae were then maintained at $28^{\circ} \mathrm{C}$ for another $24 \mathrm{~h}$. Larvae displaying no heart beat and no response to touch were regarded as dead and removed. To avoid the side effects of light period on gene expression and cold resistance, embryos and larvae were kept in dark throughout the experiment.

\section{Library construction and high-throughput sequencing}

Zebrafish larvae in the same dish were collected at $120 \mathrm{hpf}$ for RNA extraction (Figure 1A). Total RNA extraction was performed with TRIZOL reagents from Invitrogen following the manufacturer's instructions. Total RNA contents were measured using the NanoDrop 8000 from Thermo Scientific and the quality of RNA samples was assessed by agarose gel electrophoresis.

RNA library construction was then performed by Sino GenoMax Co., Ltd, Beijing, China (http://www.sinogeno $\max . \operatorname{com} /)$. Before library construction, the integrity of RNA samples was confirmed using Agilent 2100 Bioanalyzer and $4 \mu \mathrm{g}$ of total RNA was used for isolation of mRNA with Sera-mag Magnetic Oligo (dT) beads from Illumina. The purified mRNA was fragmented into small pieces (100-400 bp) using divalent cations at $94^{\circ} \mathrm{C}$ for $5 \mathrm{mi}$ nutes. Double-stranded cDNA was synthesized using the SuperScript Double-Stranded cDNA Synthesis kit (Invitrogen, Camarillo, CA) with random hexamer primers from Illumina. The synthesized cDNA was subjected to endrepair, phosphorylation, 3 ' adenylation and adapter ligation in sequential. After these steps, cDNA fragments ranging from 250 to $350 \mathrm{bp}$ were collected and purified by gel electrophoresis. The purified cDNA template was enriched by
PCR amplification and the quality of RNA library was validated in a LightCycler480 (Roche Diagnostics) using an Illumina PhiX174 Control. Three independent biological replicates for both control and cold-treated larvae were used for library construction and RNA-seq analysis. Highthroughput sequencing was performed by experts in the Analytical \& Testing Center at Institute of Hydrobiology, Chinese Academy of Sciences (http://www.ihb.ac. $\mathrm{cn} / \mathrm{fxcszx} /$ ). Multiplexed libraries were sequenced for $36 \mathrm{bp}$ at both ends using an Illumina Genome Analyzer IIx platform according to the standard Illumina protocols as reported previously [29]. The sequencing data have been deposited in NCBI Sequence Read Archive (SRA, http://www.ncbi.nlm.nih.gov/Traces/sra) and the accession number is SRA062881.

\section{Bioinformatic analysis of RNA-seq data}

The raw reads were trimmed and filtered using PRINSEQ (version 0.19.3) [74]. Low quality $(\mathrm{Q}<20)$ and ambiguous bases $(\mathrm{N})$ were first trimmed from both ends of the reads and the trimmed reads were filtered with Phred quality score ( $Q \geq 20$ for all bases) and read length ( $\geq 25 \mathrm{bp}$ ). Paired reads were extracted using cmpfastq (http://comp bio.brc.iop.kcl.ac.uk/software/cmpfastq.php). Read mapping, transcript assembly and differential expression analysis were performed according to the protocols described previously [35]. Briefly, the preprocessed reads were mapped to the genome sequence of zebrafish (Zv9.68) using TopHat (version 2.0.4) [75] with default parameters except "-segment-mismatches 1" and "-segment-length 18". The aligned reads were assembled into transcripts using Cufflinks (version 2.0.2) [76] with the following parameters "-frag-bias-correct, --multi-read-correct, --library-type fr-unstranded, --upper-quartile-norm, --totalhits-norm". The assembled transcripts were merged with the reference annotation (Danio_rerio.Zv9.68.gtf, downloaded from Ensembl) using cuffmerge. Differential expression analysis was performed using cuffdiff with the parameters "-upper-quartile-norm" and "-total-hits-norm"; the merged assembly and the fragment alignments generated by TopHat were used as input files. Calculation of mapping statistics, sorting and indexing of the read alignment files were performed using SAMtools (version 0.1.18) [77]. The mapping and assembling results were viewed via the IGVtools (version 2.1) [78].

\section{Background estimation}

To determine the background in RNA-seq analysis, 20 intergenic regions about $5 \mathrm{~kb}$ were randomly selected from each chromosome using IGVtools [78]. The selected intergenic regions were treated as exons and their FPKM values were calculated using cuffdiff as described above. 


\section{Principle component analysis (PCA)}

PCA was performed using Arraytrack [79] to elucidate the overall patterns of gene expression in the control and cold-treated samples. FPKM values of all genes identified as expressed were used for the analysis.

\section{Quantitative real time PCR (qPCR)}

qPCR analysis was performed according to the MIQE (Minimum information for publication of quantitative real-time PCR experiments) guidelines to validate the results of RNA-seq. First-strand cDNA for each sample was synthesized from $4 \mu \mathrm{g}$ of total RNA using random hexamer primer with the RevertAidTM First Strand cDNA Synthesis Kit from Fermentas. The PCR primers were designed using Primer Premier 6.0 software. qPCR was performed in a CFX Connect ${ }^{\mathrm{TM}}$ Real-Time PCR Detection System from BioRad. The amplification was carried out in a volume of $20 \mu \mathrm{L}$ containing $10 \mu \mathrm{L}$ of $2 \times$ SYBER Green Real Time PCR Master mix from TOYOBO, 2 pmol of each primer and $5 \mu \mathrm{L}$ of $10 \times$ diluted cDNA templates. Three independent biological replicates of the control and cold-treated groups were included in the analysis and all reactions were carried out in triplicates. The qPCR amplification protocol was $95^{\circ} \mathrm{C}$ for $1 \mathrm{~min}$, followed by 40 cycles of $95^{\circ} \mathrm{C}$ for $10 \mathrm{sec}, 57-60^{\circ} \mathrm{C}$ for $30 \mathrm{sec}$ (with plate read) and $72^{\circ} \mathrm{C}$ for $10 \mathrm{sec}$. After denaturized at $95^{\circ} \mathrm{C}$ for $10 \mathrm{sec}$, the melt curve of PCR product was generated by heating from $65^{\circ} \mathrm{C}$ to $95^{\circ} \mathrm{C}$ with $0.5^{\circ} \mathrm{C}$ increments and 5 sec dwell time, and a plate read at each temperature. The purity of reaction product was confirmed by the observation of a single melt peak. The amplification cycle displaying the first significant increase of the fluorescence signal was defined as threshold cycle and used for quantification (Cq).

Before qPCR analysis, the standard curve of each primer pair was generated by the regression of $\mathrm{Cq}$ values and a series of 10-fold cDNA dilutions from the mixture of all samples to be analyzed. The amplification efficiency of primers was calculated from the slope of corresponding standard curve. The sequences and amplification efficiency of primers, the accession number and official name of target genes and the length of amplicons were listed in Additional file 11. Actb1 was not found to be differentially expressed among samples by RNA-seq and therefore was used as internal reference for the normalization of gene expression. The mean normalized expression of target genes was calculated using the Q-Gene software [80].

\section{GO and KEGG pathway enrichment analyses}

Cytoscape (version 2.8.3) [81] plugins BiNGO (v.2.44) [82] and ClueGO (v.1.5) [83] were used for GO and KEGG pathway enrichment analyses, respectively. All the genes identified in this study were used as reference for the enrichment analysis. Hypergeometric test was used to identify overrepresented GO and KEGG pathway terms with a significance level at 0.05 and Beniamini \& Hochberg method was used for the correction of the p-values. The ontology and annotation files for GO enrichment analysis were downloaded from the gene ontology website (http:// www.geneontology.org/) at 08.11.2012 and the database used for KEGG pathway enrichment analysis was released on 09.11.2012.

\section{Functional clustering of differentially spliced genes}

The database for annotation, visualization and integrated discovery (DAVID) web software (v6.7, http://david.abcc. ncifcrf.gov/home.jsp) was used for the functional clustering of differentially spliced genes according to GO biological process category [84]. Gene identifier conversion was performed by g:Profiler web software (http://biit.cs.ut.ee/gpro filer/gconvert.cgi) [85].

\section{Reverse transcription PCR (RT-PCR)}

First strand cDNA samples were synthesized from DNaseI (Promega) treated total RNAs as described above. The expected amplicon size and sequences of primers used for this analysis were displayed in Additional file 10. Actb1 was used as internal control for the success of reverse transcription and PCR amplification. Amplification products were separated by electrophoresis on a $1.5 \%$ agarose gel and stained with ethidium bromide.

\section{Statistical analysis}

SPSS 15.0 software for windows was used for statistical analysis. Independent-samples t-test was performed to analyze the significant difference $(\mathrm{p}<0.05)$ in death rates between control and cold-treated larvae after exposure to $12^{\circ} \mathrm{C}$. The correlation between the data of RNA-seq and qPCR was analyzed by the Spearman's rho test.

\section{Additional files}

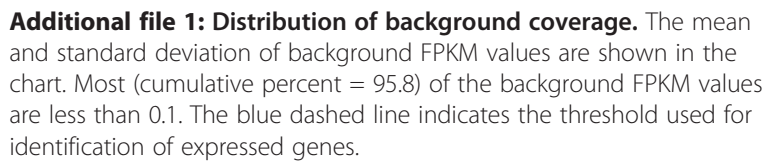

Additional file 1: Distribution of background coverage. The mean and standard deviation of background FPKM values are shown in the chart. Most (cumulative percent $=95.8$ ) of the background FPKM values are less than 0.1 . The blue dashed line indicates the threshold used for identification of expressed genes.

Additional file 2: Number of genes expressed with different abundance.

Additional file 3: Genes regulated by cold stress.

Additional file 4: A comparison between the results of microarray and RNA-seq.

Additional file 5: Enriched GO terms for cold-regulated genes.

Additional file 6: Enriched pathways for cold-regulated genes.

Additional file 7: Genes exhibited differential splicing under cold stress.

Additional file 8: Functional clustering of differentially spliced genes.

Additional file 9: Genes exhibited alternative promoter usage under cold stress. 
Additional file 10: Expression patterns of per3 transcripts during a $24 \mathrm{~h}$ light-dark cycle. (A) Expression of per3-P1 transcripts (per3-J1 and per3-J2). (B) Expression of per3-J3 (per3-P2). Zebrafish larvae were maintained under regular $12 \mathrm{~h}$ light-dark cycles from fertilization (light on at 8:00 am, light off at 8:00 pm) and exposed to cold stress $\left(16^{\circ} \mathrm{C}\right)$ from $96 \mathrm{hpf}$ to 144 hpf. The mRNA levels were detected using GPCR and the expression level relative to beta-actin was calculated using Q-gene method.

Additional file 11: Primers used for qPCR and RT-PCR.

\section{Competing interests}

The authors declare that they have no competing interests.

\section{Authors' contributions}

All the authors have read and approved the final manuscript. YL and ZC conceived the study and wrote the manuscript. $Y L$ and GS performed the data analysis. YL, YJ, GS and XH performed the experiments. GS and QL provided experimental materials.

\section{Acknowledgements}

This study was supported by the "Strategic Priority Research Program - Innovation systems of molecular module design breeding" of the Chinese Academy of Sciences and the National Natural Science Foundation of China (\#31101892 to YL). We thank Tao Li, Yan Lin and Zhixian Qiao for providing DNA library construction and RNA-seq services (Analytical \& Testing Center, IHB, CAS).

\section{Author details}

'The Key Laboratory of Aquatic Biodiversity and Conservation, Institute of Hydrobiology, Chinese Academy of Sciences, Wuhan, Hubei, PR China.

${ }^{2}$ University of the Chinese Academy of Sciences, Beijing, PR China.

Received: 28 December 2012 Accepted: 4 September 2013 Published: 11 September 2013

\section{References}

1. Wootton RJ: Fish ecology. USA: Chapman and Hall; 1992.

2. Lopez-Olmeda JF, Sanchez-Vazquez FJ: Thermal biology of zebrafish (Danio rerio). J Therm Biol 2011, 36(2):91-104.

3. Brett JR: Energetic responses of salmon to temperature - study of some thermal relations in physiology and freshwater ecology of sockeye salmon (Oncorhynchus-nerka). Am Zool 1971, 11(1):99-113.

4. Donaldson MR, Cooke SJ, Patterson DA, Macdonald JS: Cold shock and fish J Fish Biol 2008, 73(7):1491-1530.

5. Beitinger TL, Bennett WA, McCauley RW: Temperature tolerances of north American freshwater fishes exposed to dynamic changes in temperature. Environ Biol Fishes 2000, 58(3):237-275.

6. Hwang HK, Park KI, Park SW, Choi MS, Kim EO, Do JW, Oh BS: Physiologica response of juvenile red sea bream pagrus major exposed to low temperature shock. B Eur Assoc Fish Pat 2012, 32(1):34-40.

7. Kuo CM, Hsieh SL: Comparisons of physiological and biochemical responses between milkfish (Chanos chanos) and grass carp (Ctenopharyngodon idella) to cold shock. Aquaculture 2006, 251(2-4):525-536.

8. Zerai DB, Fitzsimmons KM, Collier RJ: Transcriptional response of delta-9 -desaturase gene to acute and chronic cold stress in nile tilapia, Oreochromis niloticus. J World Aquacult Soc 2010, 41(5):800-806.

9. Somero GN, Hochachka PW: Biochemical adaptation to the environment. Am Zool 1971, 11(1):159-167.

10. Guderley H: Metabolic responses to low temperature in fish muscle. Biol Rev 2004, 79(2):409-427.

11. Johnston PV, Roots BI: Brain lipid fatty acids and temperature acclimation. Comp Biochem Physiol 1964, 11:303-309.

12. Gerlach GF, Turay L, Malik KT, Lida J, Scutt A, Goldspink G: Mechanisms of temperature acclimation in the carp: a molecular biology approach. Am J Physiol 1990, 259(2 Pt 2):R237-R244.

13. Fader SC, Yu ZM, Spotila JR: Seasonal-variation in heat-shock proteins (Hsp70) in stream fish under natural conditions. J Therm Biol 1994, 19(5):335-341.

14. St-Pierre J, Charest PM, Guderley H: Relative contribution of quantitative and qualitative changes in mitochondria to metabolic compensation during seasonal acclimatisation of rainbow trout Oncorhynchus mykiss. J Exp Biol 1998, 201(21):2961-2970.
15. Gracey AY, Fraser EJ, Li W, Fang Y, Taylor RR, Rogers J, Brass A, Cossins AR: Coping with cold: an integrative, multitissue analysis of the transcriptome of a poikilothermic vertebrate. Proc Natl Acad Sci U S A 2004, 101(48):16970-16975.

16. Vergauwen $L$, Benoot D, Blust R, Knapen D: Long-term warm or cold acclimation elicits a specific transcriptional response and affects energy metabolism in zebrafish. Comp Biochem Physiol A Mol Integr Physiol 2010, 157(2):149-157.

17. Chou MY, Hsiao CD, Chen SC, Chen IW, Liu ST, Hwang PP: Effects of hypothermia on gene expression in zebrafish gills: upregulation in differentiation and function of ionocytes as compensatory responses. J Exp Biol 2008, 211(19):3077-3084.

18. Long Y, Li L, Li Q, He X, Cui Z: Transcriptomic characterization of temperature stress responses in larval zebrafish. PLOS One 2012, 7(5):e37209.

19. Ju Z, Dunham RA, Liu Z: Differential gene expression in the brain of channel catfish (Ictalurus punctatus) in response to cold acclimation. Mol Genet Genomics 2002, 268(1):87-95.

20. Podrabsky JE, Somero GN: Changes in gene expression associated with acclimation to constant temperatures and fluctuating daily temperatures in an annual killifish austrofundulus limnaeus. J Exp Biol 2004, 207(13):2237-2254

21. Kassahn KS, Crozier RH, Ward AC, Stone G, Caley MJ: From transcriptome to biological function: environmental stress in an ectothermic vertebrate, the coral reef fish Pomacentrus moluccensis. BMC Genomics 2007, 8:358.

22. Vornanen M, Hassinen M, Koskinen H, Krasnov A: Steady-state effects of temperature acclimation on the transcriptome of the rainbow trout heart. Am J Physiol Regul Integr Comp Physiol 2005, 289(4):R1177-R1184.

23. Green BS, Fisher R: Temperature influences swimming speed, growth and larval duration in coral reef fish larvae. J Exp Mar Biol Ecol 2004, 299(1):115-132.

24. Beitinger TL, Bennett WA: Quantification of the role of acclimation temperature in temperature tolerance of fishes. Environ Biol Fishes 2000, 58(3):277-288

25. Kelty JD, Lee RE: Induction of rapid cold hardening by cooling at ecologically relevant rates in Drosophila melanogaster. I Insect Physiol 1999, 45(8):719-726.

26. Clark MS, Worland MR: How insects survive the cold: molecular mechanisms-a review. J Comp Physiol B Biochem Syst Environ Physiol 2008, 178(8):917-933.

27. Cortemeglia C, Beitinger TL: Temperature tolerances of wild-type and red transgenic zebra danios. Trans Am Fish Soc 2005, 134(6):1431-1437.

28. Wang Z, Gerstein M, Snyder M: RNA-Seq: a revolutionary tool for transcriptomics. Nat Rev Genet 2009, 10(1):57-63.

29. Wang ZY, Fang BP, Chen JY, Zhang XJ, Luo ZX, Huang LF, Chen XL, Li YJ: De novo assembly and characterization of root transcriptome using illumina paired-end sequencing and development of CSSR markers in sweetpotato (Ipomoea batatas). BMC Genomics 2010, 11:726.

30. Rino J, Carmo-Fonseca M: The spliceosome: a self-organized macromolecular machine in the nucleus? Trends Cell Biol 2009, 19(8):375-384.

31. Will CL, Luhrmann R: Spliceosome structure and function. Cold Spring Harb Perspect Biol 2011, 3:a003707.

32. Hoskins AA, Moore MJ: The spliceosome: a flexible, reversible macromolecular machine. Trends Biochem Sci 2012, 37(5):179-188.

33. Fromont-Racine M, Senger B, Saveanu C, Fasiolo F: Ribosome assembly in eukaryotes. Gene 2003, 313:17-42.

34. Perez-Fernandez J, Roman A, De Las RJ, Bustelo XR, Dosil M: The $90 \mathrm{~S}$ preribosome is a multimodular structure that is assembled through a hierarchical mechanism. Mol Cell Biol 2007, 27(15):5414-5429.

35. Trapnell C, Roberts A, Goff L, Pertea G, Kim D, Kelley DR, Pimentel H, Salzberg SL, Rinn JL, Pachter L: Differential gene and transcript expression analysis of RNA-seq experiments with TopHat and cufflinks. Nat Protoc 2012, 7(3):562-578.

36. Delaunay F, Thisse $C$, Thisse $B$, Laudet $\mathrm{V}$ : Differential regulation of period 2 and period 3 expression during development of the zebrafish circadian clock. Gene Expr Patterns 2003, 3(3):319-324.

37. Schaefer J, Ryan A: Developmental plasticity in the thermal tolerance of zebrafish Danio rerio. J Fish Biol 2006, 69(3):722-734.

38. Scott GR, Johnston IA: Temperature during embryonic development has persistent effects on thermal acclimation capacity in zebrafish. Proc Natl Acad Sci U S A 2012, 109(35):14247-14252. 
39. Overgaard J, Malmendal A, Sorensen JG, Bundy JG, Loeschcke V, Nielsen NC, Holmstrup M: Metabolomic profiling of rapid cold hardening and cold shock in Drosophila melanogaster. J Insect Physiol 2007, 53(12):1218-1232.

40. Alderman SL, Klaiman JM, Deck CA, Gillis TE: Effect of cold acclimation on troponin I isoform expression in striated muscle of rainbow trout. Am J Physiol Regul Integr Comp Physiol 2012, 303(2):R168-R176.

41. Chen RE, Thorner J: Function and regulation in MAPK signaling pathways: lessons learned from the yeast Saccharomyces cerevisiae. Biochimica Et Biophysica Acta-Molecular Cell Research 2007, 1773(8):1311-1340.

42. Colcombet J, Hirt H: Arabidopsis MAPKs: a complex signalling network involved in multiple biological processes. Biochem J 2008, 413:217-226.

43. Obata T, Brown GE, Yaffe MB: MAP kinase pathways activated by stress: the p38 MAPK pathway. Crit Care Med 2000, 28(4):N67-N77.

44. Siliciano JD, Canman CE, Taya Y, Sakaguchi K, Appella E, Kastan MB: DNA damage induces phosphorylation of the amino terminus of p53. Genes Dev 1997, 11(24):3471-3481.

45. Graeber TG, Peterson JF, Tsai M, Monica K, Fornace AJ, Giaccia AJ: Hypoxia induces accumulation of $P 53$ protein, but activation of a $G(1)$-phase checkpoint by Low-oxygen conditions is independent of P53 status. Mol Cell Biol 1994, 14(9):6264-6277.

46. Lakin ND, Jackson SP: Regulation of p53 in response to DNA damage. Oncogene 1999, 18(53):7644-7655.

47. Ren YS, Sun CB, Sun Y, Tan HB, Wu YC, Cui B, Wu ZG: PPAR gamma protects cardiomyocytes against oxidative stress and apoptosis via $\mathrm{Bcl}-2$ upregulation. Vasc Pharmacol 2009, 51(2-3):169-174.

48. Blanquicett C, Kang BY, Ritzenthaler JD, Jones DP, Hart CM: Oxidative stress modulates PPAR gamma in vascular endothelial cells. Free Radic Biol Med 2010, 48(12):1618-1625.

49. Mastrangelo AM, Marone D, Laido G, De Leonardis AM, De Vita P: Alternative splicing: enhancing ability to cope with stress via transcriptome plasticity. Plant Sci 2012, 185:40-49.

50. Ali GS, Reddy ASN: Regulation of alternative splicing of Pre-mRNAs by stresses. Curr Top Microbiol Immunol 2008, 326:257-275.1.

51. Dutertre M, Sanchez G, Barbier J, Corcos L, Auboeuf D: The emerging role of pre-messenger RNA splicing in stress responses sending alternative messages and silent messengers. RNA Biol 2011, 8(5):740-747.

52. Keren H, Lev-Maor G, Ast G: Alternative splicing and evolution: diversification, exon definition and function. Nat Rev Genet 2010, 11(5):345-355.

53. Takechi H, Hosokawa N, Hirayoshi K, Nagata K: Alternative 5 ' splice-site selection induced by heat-shock. Mol Cell Biol 1994, 14(1):567-575.

54. Fujikake N, Nagai Y, Popiel HA, Kano H, Yamaguchi M, Toda T: Alternative splicing regulates the transcriptional activity of Drosophila heat shock transcription factor in response to heat/cold stress. FEBS Lett 2005, 579(17):3842-3848.

55. Guinea B, Ligos JM, de Lera TL, Martin-Caballero J, Flores J, de la Pena MG, Garcia-Castro J, Bernad A: Nucleocytoplasmic shuttling of STK16 (PKL12), a golgi-resident serine/threonine kinase involved in VEGF expression regulation. Exp Cell Res 2005, 312(2):135-144.

56. Wang XL, Yan DW, Teng MJ, Fan JW, Zhou CZ, Li DW, Qiu GQ, Sun X, Li T, Xing $\mathrm{TH}$, et al: Reduced expression of PER3 is associated with incidence and development of colon cancer. Ann Surg Oncol 2012, 19(9):3081-3088,

57. Im JS, Jung BH, Kim SE, Lee KH, Lee JK: Per3, A circadian gene, is required for Chk2 activation in human cells. FEBS Lett 2010, 584(23):4731-4734.

58. Van der Veen DR, Dijk DJ, Archer SN: A role for PERIOD3 in sleep/wake rhythms: photic responses in humanised knock-in mice and gene expression correlates of PER3 expression. J Sleep Res 2012, 21:333-333.

59. Bass J, Takahashi JS: Circadian integration of metabolism and energetics. Science 2010, 330(6009):1349-1354.

60. Lee $Y$, Montell C: Drosophila TRPA1 functions in temperature control of circadian rhythm in pacemaker neurons. J Neurosci 2013, 33(16):6716-6725.

61. Lahiri K, Vallone D, Gondi SB, Santoriello C, Dickmeis T, Foulkes NS: Temperature regulates transcription in the zebrafish circadian clock. PLOS Biol 2005, 3(11):2005-2016.

62. Vatine $G$, Vallone $D$, Gothilf $Y$, Foulkes NS: It's time to swim! zebrafish and the circadian clock. FEBS Lett 2011, 585(10):1485-1494.

63. Martino-Catt S, Ort DR: Low temperature interrupts circadian regulation of transcriptional activity in chilling-sensitive plants. Proc Natl Acad Sci U S A 1992, 89(9):3731-3735.

64. Bieniawska Z, Espinoza C, Schlereth A, Sulpice R, Hincha DK, Hannah MA: Disruption of the Arabidopsis circadian clock is responsible for extensive variation in the cold-responsive transcriptome. Plant Physio/ 2008, 147(1):263-279.

65. Hofmann NR: Alternative splicing links the circadian clock to cold tolerance. Plant Cell 2012, 24(6):2238-2238.

66. James AB, Syed NH, Bordage S, Marshall J, Nimmo GA, Jenkins Gl, Herzyk P, Brown JWS, Nimmo HG: Alternative splicing mediates responses of the Arabidopsis circadian clock to temperature changes. Plant Cell 2012, 24(3):961-981.

67. Seo PJ, Park MJ, Park CM: Alternative splicing of transcription factors in plant responses to low temperature stress: mechanisms and functions. Planta 2013, 237(6):1415-1424.

68. Lackner DH, Schmidt MW, Wu S, Wolf DA, Bahler J: Regulation of transcriptome, translation, and proteome in response to environmental stress in fission yeast. Genome Biol 2012, 13(4):R25

69. Dobson CM: Protein folding and misfolding. Nature 2003, 426(6968):884-890.

70. Dias CL, Ala-Nissila T, Wong-ekkabut J, Vattulainen I, Grant M, Karttunen M: The hydrophobic effect and its role in cold denaturation. Cryobiology 2010, 60(1):91-99.

71. Guo M, Xu Y, Gruebele M: Temperature dependence of protein folding kinetics in living cells. Proc Natl Acad Sci U S A 2012, 109(44):17863-17867.

72. Sali A, Shakhnovich E, Karplus M: How does a protein fold. Nature 1994, 369(6477):248-251

73. Long Y, Li Q, Zhong S, Wang Y, Cui Z: Molecular characterization and functions of zebrafish $A B C C 2$ in cellular efflux of heavy metals. Comp Biochem Physiol C Toxicol Pharmacol 2011, 153(4):381-391.

74. Schmieder R, Edwards R: Quality control and preprocessing of metagenomic datasets. Bioinformatics 2011, 27(6):863-864.

75. Trapnell C, Pachter L, Salzberg SL: TopHat: discovering splice junctions with RNA-Seq. Bioinformatics 2009, 25(9):1105-1111.

76. Trapnell C, Williams BA, Pertea G, Mortazavi A, Kwan G, van Baren MJ, Salzberg SL, Wold BJ, Pachter L: Transcript assembly and quantification by RNA-Seq reveals unannotated transcripts and isoform switching during cell differentiation. Nat Biotechnol 2010, 28(5):511-515.

77. Li H, Handsaker B, Wysoker A, Fennell T, Ruan J, Homer N, Marth G, Abecasis G, Durbin R: The sequence alignment/Map format and SAMtools. Bioinformatics 2009, 25(16):2078-2079.

78. Thorvaldsdottir H, Robinson JT, Mesirov JP: Integrative genomics viewer (IGV): high-performance genomics data visualization and exploration. Brief Bioinform 2013, 14(2):178-192.

79. Fang H: The FDA genomics tool - ArrayTrack. Mol Cell Toxicol 2009, 5(3):40-40.

80. Muller PY, Janovjak H, Miserez AR, Dobbie Z: Processing of gene expression data generated by quantitative real-time RT-PCR. Biotechniques 2002, 32(6):1372-1374. 1376, 1378-1379.

81. Shannon P, Markiel A, Ozier O, Baliga NS, Wang JT, Ramage D, Amin N, Schwikowski B, Ideker T: Cytoscape: a software environment for integrated models of biomolecular interaction networks. Genome Res 2003, 13(11):2498-2504.

82. Maere S, Heymans K, Kuiper M: BiNGO: a cytoscape plugin to assess overrepresentation of gene ontology categories in biological networks. Bioinformatics 2005, 21(16):3448-3449.

83. Bindea G, Mlecnik B, Hackl H, Charoentong P, Tosolini M, Kirilovsky A, Fridman WH, Pages F, Trajanoski Z, Galon J: ClueGO: a cytoscape plug-in to decipher functionally grouped gene ontology and pathway annotation networks. Bioinformatics 2009, 25(8):1091-1093.

84. Huang DW, Sherman BT, Tan Q, Collins JR, Alvord WG, Roayaei J, Stephens R, Baseler MW, Lane HC, Lempicki RA: The DAVID gene functional classification tool: a novel biological module-centric algorithm to functionally analyze large gene lists. Genome Biol 2007, 8(9):R183.

85. Reimand J, Kull M, Peterson H, Hansen J, Vilo J: g : profiler - a web-based toolset for functional profiling of gene lists from large-scale experiments. Nucleic Acids Res 2007, 35:W193-W200.

doi:10.1186/1471-2164-14-612

Cite this article as: Long et al:: Transcriptomic characterization of cold acclimation in larval zebrafish. BMC Genomics 2013 14:612 